\title{
Acerca de algunos factores condicionantes del abordaje bioético en psiquiatría.
}

\begin{abstract}
About some determinants of the bioethical approach in psychiatry. Juan Carlos Stagnaro ${ }^{1}$

RESUMEN

En las últimas décadas la bioética ha penetrado la psiquiatría más tardíamente que en otras especialidades médicas, encontrándose en las prácticas y en la preceptiva de diversas declaraciones internacionales, así como en una creciente bibliografía sobre el tema, abundante opiniones acerca de esta problemática. En este artículo se pasa revista a ciertos tópicos que pueden constituirse en fuentes institucionales, epistemológicas y socioeconómicas de problemáticas bioéticas, tales como los efectos del reduccionismo biomédico en la psiquiatría y los aspectos del diagnóstico que pueden ser vehículo de problemas éticos.
\end{abstract}

PALABRAS CLAVE: Bioética y psiquiatría, códigos éticos en psiquiatría, reduccionismo biomédico y psiquiatría, sistemas de salud.

\section{SUMMARY}

In the last decades, later than other medical specialties, bioethics has penetrated psychiatry and many points of view can be found on this issue in the practice and provisions included in several international declarations, as well as in an increasing related literature. This article carefully reviews certain topics that can become institutional, epistemological, and socioeconomic sources of bioethical concerns, such as the effects of biomedical reductionism in psychiatry and diagnostic aspects which can lead to ethical issues.

KEYWORDS: Bioethics and psychiatry, codes of ethics on psychiatry, biomedical reductionism and psychiatry, health systems.

\section{INTRODUCCIÓN}

"Como profesionales de la medicina, los psiquiatras deben ser conscientes de las implicaciones éticas que se derivan del ejercicio de su profesión y de las exigencias éticas específicas de la especialidad en psiquiatría. Como miembros de la sociedad, los psiquiatras deben luchar por un tratamiento justo y equitativo de los enfermos mentales, en aras de una justicia social igual para todos".

\section{De la Declaración de Madrid, Asociación Mundial} de Psiquiatría
A partir de los años setenta del siglo XX comenzó a delinearse una incipiente vinculación entre la bioética y la psiquiatría. Este fenómeno ocurrió en cierta conexión contemporánea con los cambios introducidos por las nuevas opciones terapéuticas brindadas por la psicofarmacología, la crítica a las viejas instituciones de internación "manicomial", la clínica de la rehabilitación y reinserción social de los enfermos de larga evolución y los nuevos enfoques y conceptos preventivos y comunitarios provenientes de la perspectiva que inauguró el denominado campo de la Salud Mental en la etapa posterior a la Segunda Guerra Mundial.

1 Profesor Regular Titular, Departamento de Psiquiatría y Salud Mental. Docente e investigador, Departamento de Humanidades Médicas. Facultad de Medicina, Universidad de Buenos Aires. Buenos Aires, Argentina.

Editor Jefe de Vértex, Revista Psiquiátrica Argentina 
Sin embargo la relación entre ambas disciplinas fue, y sigue siendo, lenta y laboriosa en comparación con el interés que despertó la bioética en otros ámbitos de la medicina $(1,2)$; en consecuencia, la penetración de conceptos bioéticos en relación al diagnóstico y a la terapéutica en psiquiatría ha sido escasa (3-5). Quizás más motivados por conflictos candentes aparecidos en otras especialidades médicas en las que se plantearon dilemas éticos originales $(1,5-7)$, los bioeticistas se interesaron menos en la psiquiatría y en los problemas que ésta pudiera presentarles como objeto de estudio (8).

Cabría atribuir también dicha diferencia a múltiples motivos ligados al objeto y a los recursos terapéuticos de la psiquiatría. En efecto, es probable que la bioética, que vino a poner un marco de reflexión y consenso a la (omni)potente tecnología biomédica, haya encontrado escasos argumentos para interpelar a la artesanal terapéutica psiquiátrica. Por su lado, y a diferencia de los médicos de otras especialidades, a los psiquiatras no les ha sido fácil incorporar criterios de acción provenientes de la reflexión bioética en razón de las dificultades que presenta su disciplina para ajustarse a las exigencias del modelo biomédico contemporáneo -en el que predomina una manera objetivante, a-histórica y a-social de pensar respecto a salud y enfermedad (9)- y/o a las de la metodología científica.

Este conflicto, ya conocido desde hace tiempo, deriva, principalmente, aunque no en forma exclusiva, de las características específicas del "saber psiquiátrico", originado, más que ninguna otra especialidad médica, en la encrucijada de la biología, la psicología, la antropología y la filosofía (10). No es que el resto de la medicina esté exenta, a fin de acceder a una praxis de excelencia, de la necesidad de integrar en el "arte de curar" nociones y acciones provenientes de todas esas disciplinas; lo que sucede es que la psiquiatría siempre gravitó en una posición marginal y excéntrica respecto de aquel modelo biomédico. De otro lado y por múltiples razones, la psiquiatría requeriría un particular atravesamiento de la perspectiva bioética al confrontar nuevas y particulares condiciones derivadas de las coordenadas epistemológicas, históricas y sociales que atraviesan a la medicina y el campo de la Salud en su conjunto $(11,12)$.

Finalmente, no debe olvidarse que a la psiquiatría le caben también las problemáticas bioéticas generales de la medicina que no trataremos aquí, aunque han sido objeto de comunicaciones y estudios suficientemente conocidos. Tales son, entre muchas, las implicancias del diagnóstico $(13,14)$, las particularidades del consentimiento informado, altamente complejo en la especialidad $(15,16)$, el secreto médico (17) la confidencialidad (18), la información al paciente sobre los alcances y efectos de la terapéutica (19-25), la ética en la psiquiatría comunitaria, (26) la vinculación de los médicos con la industria farmacéutica, el espinoso problema del peritaje psiquiátrico judicial (27-29), etc.

En este artículo nos limitaremos a presentar algunas reflexiones acerca de ciertas áreas de conflicto que tienen una particular importancia en el ejercicio cotidiano de la especialidad.

\section{La preceptiva internacional y las publicaciones sobre bioética y psiquiatría}

Existe una variada preceptiva internacional de códigos éticos en psiquiatría. Recién en 1973 se elaboró el primer código específico para la psiquiatría, por parte de la American Psychiatric Association (APA) tomando como antecedente el de la American Medical Association (AMA). De igual manera, la Canadian Psychiatric Association se basó en el código de Ética de la Canadian Medical Association para elaborar el suyo añadiendo comentarios, como lo hizo la APA. Finalmente, la Asociación Mundial de Psiquiatría (WPA) celebró en 1976 su primer congreso sobre aspectos éticos de la psiquiatría, en Londres. Clarece Blomquist, profesor de Ética Médica en el Instituto Karolinska, propuso la necesidad de un propio código ético. Su aporte inspiró el documento introducido un año más tarde en el Congreso Mundial de la WPA en Honolulu. Ese pronunciamiento que se conoce como Declaración de Hawai recoge las pautas consideradas como los requisitos éticos mínimos en psiquiatría. Basada en una ética del deber, insta al psiquiatra a servir a los mejores intereses del paciente, ofrecer el mejor tratamiento disponible, obtener consentimiento informado antes de efectuar un procedimiento o un tratamiento, y aplicar la experiencia y los conocimientos profesionales sólo con legítimos propósitos diagnósticos y terapéuticos. También exige el respeto a la confidencialidad y la obtención de consentimiento informado para incluir al paciente en actividades docentes y señala una serie de circunstancias que el psiquiatra deberá tener en cuenta: la enfermedad mental puede convertir al paciente en incompetente para prestar consentimiento informado; la psiquiatría puede ser utilizada perversamente por 
los poderes políticos o de otro tipo; en psiquiatría pueden existir relaciones no terapéuticas, como es el caso de los peritajes, etc. Aunque muy importante, no ha sido suficientemente incorporada a los estatutos de los organismos psiquiátricos nacionales. Los posibles objetivos principales de los códigos de Ética en Psiquiatría giran en torno a los siguientes tópicos (30):

1. Proteger y promocionar el estatus profesional de los psiquiatras.

2. Formar parte intrínseca de los procesos de autorregulación de la profesión.

3. Sensibilizar a los psiquiatras respecto a la dimensión ética de su trabajo.

4. Servir de herramienta en la educación profesional.

Por su lado, la cantidad y difusión de publicaciones sobre un tema en la literatura científica pueden constituir un instrumento de medida de la relevancia que ese tema tiene en el seno de una comunidad profesional.

En los últimos años, diversos autores han publicado sendos trabajos de conjunto intentando aplicar principios bioéticos en el campo de la psiquiatría y la Salud Mental (4,11,12,30-35). Recientemente, se dio a conocer un estudio bibliométrico con diseño observacional retrospectivo y cobertura dentro de la base de datos Web of Sciences (WoS, Thomas Reuters); en él se cuantificó la cantidad de publicaciones a nivel internacional sobre temas que relacionaban Bioética y Salud Mental en el período 2002-2011. A tal fin los autores trabajaron con los registros indizados en la $W o S$, a fin de estudiar la visibilidad y repercusión científica (mediante el análisis de citas recibidas), identificar las revistas científicas. Los autores realizaron el estudio bibliométrico basándose en los artículos sobre Bioética y Salud Mental publicados en las siguientes revistas: Acta Bioethica, Australian and New Zealand Journal of Psychiatry, Revue Canadienne de Psychiatrie, Psychologie canadienne, Current Opinion in Psychiatry, Ethics and Behavior, Journal of Bioethics Inquiry, Journal of Forensic Psychiatry and Psychology, Journal of Medical Ethics, Journal of Psychiatry and Mental Health Nursing, Journal of de American Academy of Child and Adolescent Psychiatry, Psychiatric Clinics of North America, que han generado más producción en la materia ( $\geq 3$ documentos) y su Factor de Impacto (FI) 2010 en los Journal Citation Reports ${ }^{\circledR}(\mathrm{JCR})$ y los principales temas tratados mediante un análisis de contenido de los títulos, resúmenes y palabras clave presentadas en dichos documentos (36); se obtuvieron así 159 documentos (118 artículos de revista, 7 editoriales, 20 artículos de revisión y 14 actas de congresos). Según los autores se puede concluir que: 1) "existe una escasa producción científica que conjugue temas de Bioética y Salud Mental, aunque ésta tiene una repercusión científica y visibilidad que pueden considerarse aceptables y 2) dicha producción científica aparece dispersa en un elevado número de publicaciones y categorías temáticas, lo que sugiere una falta de solidez editorial a la hora de abordar la temática Bioética-Salud Mental en las publicaciones internacionales más representativas, así como una carencia de líneas de investigación consolidadas" (36).

Entre los trabajos publicados en este trabajo he tomado en cuenta solo de algunos de los más significativos, especialmente en lengua castellana, y la preocupación se centró, principalmente, en el diagnóstico psiquiátrico como problema ético, el consentimiento informado en psiquiatría -previo a la aplicación de pruebas diagnósticas o tratamientos determinados- $(37,38)$, la valoración de la capacidad de decisión (competencia) de los pacientes $(39,40)$, los aspectos éticos de la terapéutica psiquiátrica, (especialmente la legitimidad de la psicoterapia, por un lado y el abuso del tratamiento farmacológico, por otro, en cuanto se admite que tanto la psicoterapia como el psicoterapeuta, así como la prescripción medicamentosa, son todo menos neutrales, en relación a los valores implicados en ellas), los de la rehabilitación del paciente mental y los dilemas éticos que conlleva la elaboración de los informes psiquiátricos, la confidencialidad que debe estar presente a lo largo de todo el proceso asistencial $(41,42)$, el documento de voluntad anticipada (habitualmente mencionado en relación con el "final de la vida"; pero que puede ser aplicado "durante la vida") en el ámbito psiquiátrico (43-50) y los derechos humanos del enfermo mental, que han dado lugar a diversos códigos o declaraciones nacionales e internacionales en relación a la especialidad $(51,52)$. También ha aparecido una creciente reflexión en la última década sobre los efectos éticos del desarrollo de la neurobiología sobre la psiquiatría: la neuroética (53-59).

En el plano institucional las actividades de la bioética tomaron cuerpo, en los años '70, con la creación en los EE. UU. de los Comités de Bioética (asistencial de investigación) bajo la forma de grupos multidisciplinarios cuyo objetivo ha sido la protección de los derechos y el cuidado de las personas en el seno de las instituciones de salud (60). 


\section{Fuentes institucionales, epistemológicas y socioeconómicas de problemáticas bioéticas}

\section{a) La atención psiquiátrica}

Un aspecto de importancia esencial, causante de problemáticas tributarias de la bioética, es el tratamiento que se reserva a los enfermos mentales en la sociedad. El asilo para locos, la casa de dementes, el manicomio, el hospicio u hospital para enfermos mentales, o como quiera que se lo designara durante el siglo XIX, contribuyó, como ninguna otra institución sanitaria respecto de las otras especialidades médicas, a la definición del rol del psiquiatra y de la psiquiatría como especialidad médica (62).

En cada etapa de transformación de la medicina mental la evolución social y los cambios en la percepción de las enfermedades mentales en el seno de la sociedad occidental así como en las teorías, las instituciones psiquiátricas y el rol del psiquiatra, se conservan aspectos heredados desde el origen de la especialidad, articulados con efectos de innovaciones introducidas por la evolución histórica (63). Por otro lado, empujados por la inercia administrativa y por concepciones retrógradas respecto de las afecciones mentales persisten formas de atención que permiten la subsistencia de instituciones manicomiales, verdaderos depósitos de personas hacinadas y desposeídas de los derechos más elementales de acceso a una vida digna.

Esa situación afecta, fundamentalmente en los países en vías de desarrollo (aunque también en forma creciente en algunos países centrales como consecuencia de los recortes en los presupuestos sociales ante la crisis financiera internacional), a los pacientes pobres, quienes no tienen los medios para pagar tratamientos que se conocen y son efectivos, y que quedan reservados, por lo tanto, a los sectores sociales más pudientes. Como resultado de ello se puede hablar de una psiquiatría del privilegio social y otra de la miseria económica, lo que constituye para la medicina un verdadero baldón. Ligada a este fenómeno se debe considerar la particular situación de los enfermos mentales frente a la ley y las derivaciones de ella respecto de las normativas de internación psiquiátrica, a la declaración de insania, etc.; estos aspectos violan claramente nociones de solidaridad social, de derechos humanos de los pacientes y de sus libertades individuales $(64,65)$.

En el momento actual, imprescindibles transformaciones en la atención psiquiátrica deben ocupar el centro de la escena de la especialidad. El modelo del viejo hospital psiquiátrico manicomial ya no se puede sostener (62). No existen razones médicas, técnicas ni económicas que lo justifiquen. La psiquiatría contemporánea a nivel internacional (véase la Declaración de Caracas (66) por ejemplo), recomienda terminar con esa forma de hospitalización por el carácter deletéreo que tiene sobre los enfermos mentales y, por el contrario, aconseja el tratamiento en forma ambulatoria, en instituciones adecuadas y en la comunidad, para evitar el desarraigo, el hospitalismo y la anomia consecuentes a las prolongadas internaciones en los hospitales psiquiátricos tradicionales (38).

Quede claro que instituciones psiquiátricas especializadas, con políticas de admisiones cortas para la atención de pacientes aquejados por trastornos psiquiátricos severos o en crisis que no pueden ser tratadas en los servicios de Psicopatología de los hospitales generales y que, por lo tanto, representan con frecuencia riesgo para sí o para terceros, siguen siendo necesarias. Sin embargo, entre otras características, su tamaño (número de pacientes que alberguen) y la cualidad o naturaleza intensiva de los tratamientos administrados deben ser condiciones sine qua non de su funcionamiento. Los Servicios de Salud Mental en los hospitales generales, la atención en Centros de Salud, y en las instituciones intermedias en la comunidad (Hospitales de Día, Casas a medio camino, Casas de convivencia, etc.) son, a tal efecto, efectores complementarios válidos para disminuir aún más la necesidad de los centros psiquiátricos monovalentes. Aun cuando esta propuesta es racional y razonable, se verifica una particular inercia del sistema de atención e, inclusive, en algunos países en los que la situación había evolucionado hacia sistemas más adecuados, se observa hoy una involución.

Causas corporativas, jurídicas, culturales y, sobre todo, más económico-políticas que científicas pueden explicar esos fenómenos (por ejemplo: el debilitamiento de la política del Sector en Francia, el fenómeno de los homeless o "sin techo", la diferente calidad de la reforma psiquiátrica entre el norte (rico) y el sur (pobre) de Italia, la progresiva judicialización de pacientes mentales en muchos países europeos, la falta de reformas que superen las estructuras manicomiales en los países en desarrollo, etc.). Es así que resulta necesario "analizar más de cerca los conceptos que, cargados de un fardo ideológico frecuentemente no explicitado, tienden a demonizar al hospital psiquiátrico [...] clamando por su desaparición lisa y llana, sin proponer una 
estructura de reemplazo, así como también aquéllos que lo defienden como efector único de un sistema de salud anticuado y hospitalocéntrico con el inconfesado interés de defender intereses corporativos, de pequeños grupos de poder o personales" (62). Estos fenómenos que engloban el destino de los enfermos mentales en nuestra sociedad también merecen ser estudiados desde la perspectiva bioética.

\section{b) Una disciplina en busca de su especificidad}

La complejidad de los desafíos que enfrenta la psiquiatría contemporánea no se agota en la situación institucional y sanitaria. Simultáneamente con el mantenimiento del viejo hospital asilar como estructura central del dispositivo psiquiátrico, las fronteras de la especialidad y sus pertinencias se orientaron en el último cuarto de siglo en múltiples direcciones (67).

La psiquiatría se ha ido extendiendo en la cultura y su expansión sobre las más diversas conductas humanas -cuyo beneficio puede encontrarse en la mayor atención a la salud mental poblacional- también tiene meandros que se infiltran, en esa tenue y opinable frontera de la patología con las más diversas manifestaciones de la "pena de vivir", y conducen al riesgo de proporcionar una "coartada" basada en el discurso de la medicina mental, a formas de malestar social cuyas motivaciones están muy lejos de constituir el objeto de la psiquiatría como especialidad médica.

Por otro lado, las teorías explicativas de los trastornos mentales se han multiplicado y se atraviesa una crisis del paradigma psiquiátrico.

\section{c) El reduccionismo biomédico en psiquiatría}

La expresión del cientificismo contemporáneo en el campo de la medicina es la corriente reduccionista biomédica, hoy hegemónica. Al desdeñar la reflexión crítica aportada por la valoración ética de los actos médicos, la mentalidad cientificista tiende a sostener la idea de que lo técnicamente posible es, por ello mismo, moralmente admisible.

La perspectiva antropológica en la tarea médica ha perdido influencia ante el poder que la tecnología ha otorgado, en las últimas décadas, al diagnóstico y a la terapéutica (68). Los logros alcanzados por esa vía no se han integrado con una concepción holística del paciente lo cual conduce, con frecuencia, a un desvanecimiento de la condición humana detrás de las estadísticas y los criterios mecanicistas biológicos de normalidad y salud. Como saldo de tanta excelencia científica, el profesional, en especial entre los más jóvenes, es inducido a desconocer la perspectiva humanística de la labor médica $(69,70)$ y queda librado a su propio albedrío frente a la inmensa cantidad de interrogantes que se les plantean en la práctica profesional, al no adquirir durante su formación, las herramientas críticas para resolver tales dificultades (69). Esta impronta cientificista en la sociedad en general, y en la medicina en particular, está siendo cuestionada desde diversas vertientes al entenderse que solamente habrá una sociedad más humana si se otorga mayor importancia a la ética sobre la técnica y a las personas sobre los objetos $(9,71)$.

Las Facultades de Medicina, en niveles de grado y postgrado, y las organizaciones científicas, no escapan a esa polémica. Una de sus temas más prometedores es la creciente tendencia a incorporar la perspectiva bioética en la formación de los médicos generales y de los especialistas.

Sin embargo, como se dijo antes, deben reconocerse dificultades intrínsecas al saber psiquiátrico contemporáneo. Tomar decisiones con perspectiva bioética implica hacerlo en el marco de conocimientos posibles que -y aquí reside uno de los problemas importantes- condicionarán dichas opciones éticas; más aún, podrían prefigurarlas. En las condiciones actuales, esos conocimientos provienen en amplios sectores de la psiquiatría contemporánea de una estructura de pensamiento biomédico que fácilmente se desliza a una posición reduccionista, $y$, por ende, las decisiones que se tomen estarán viciadas del sesgo epistemológico provisto por esa matriz de pensamiento (69).

Cabe entonces avanzar una posición para la polémica: la única posibilidad de encarar una medicina atravesada por un ética respetuosa del sufrimiento humano debe partir de una crítica radical del modelo reduccionista biomédico. Y la única posibilidad de hacerlo se puede encontrar en la actualización de lo que ha dado en llamarse el modelo médico antropológico o sea una medicina de la persona, considerada ésta en su integridad e individualidad $(72,73)$. El complemento de ese paciente es el médico antropológico, también denominado médico integral (74). Solamente desde esa formación y con esa concepción de la relación médico-paciente se hará prácticamente posible una toma en consideración de las problemáticas éticas de la persona en su "integridad e individualidad" (72). 
La psiquiatría constituye un caso particular entre las especialidades médicas y, en consecuencia, se ubica en una suerte de encrucijada epistemológica, en la que se articulan nociones propias de diversos campos disciplinares de las ciencias humanas y naturales. Esta particular mixtura que, por un lado constituye su riqueza y la convierte en "la más humanista de las especialidades médicas", por otro, la aleja del modelo biomédico imperante que exige estándares de "cientificidad" hipotético-deductivos, difícilmente alcanzables por ella. La dimensión intersubjetiva en el diagnóstico, con su extraordinariamente rica pero no reproducible carga hermenéutica, los límites de la investigación fisiopatológica generados por la casi imposibilidad de arribar de la investigación en animales a conclusiones extrapolables al nivel humano, la dificultad para diseñar modelos neurales incruentos de estudio científico del comportamiento normal y patológico en el hombre (hoy abordado con las técnicas de neuroimágenes), los obstáculos del establecimiento de protocolos válidos para la investigación en psicoterapias, la dosis de empirismo que conlleva el diseño de estrategias terapéuticas, la incertidumbre en el pronóstico evolutivo de muchas afecciones mentales, constriñen a la psiquiatría a operar en un campo que adolece de criterios nosológicos estrictos y esquemas terapéuticos protocolizados, como pueden exhibir otras especialidades de la medicina. Por ende, su situación, que podría considerarse envidiable en cuanto a su riqueza de inspiración multidisciplinaria para aproximarse, comprender y acudir en ayuda del ser humano enfermo, plantea al mismo tiempo dificultades para establecer los límites de su campo de pertinencia y para delimitar con nitidez el objeto de su praxis.

En procura de resolver esos problemas y obtener su legitimación en el seno de la profesión médica, basándose en criterios biológicos, los psiquiatras han logrado, a lo largo de los dos siglos de desarrollo de la especialidad, dar consistencia a un "núcleo duro" de la misma en torno a ciertas afecciones de probada base orgánica cerebral capaces de alterar el comportamiento (demencias, ciertas formas de retraso mental, trastornos psicóticos de origen orgánico, entre otros), a los cuales se asimilaron las principales formas de los antiguamente llamados trastornos endógenos (esquizofrenia, depresiones severas, trastornos bipolares) a los que se les atribuye una probada (aunque siempre discutida) base fisiopatológica reforzada en los últimos años por estudios científicos neurobiológicos (genéticos, de neuroimágenes, farmacológicos, etc.).
En esa línea de pensamiento la especialidad, alejándose de la orientación psicodinámica (particularmente representada por las corrientes psicoanalíticas y fenomenológicas que tuvieron una fuerte presencia en la psicopatología a lo largo de casi todo el siglo $\mathrm{XX}$ ) ha sufrido una transformación en su orientación teórica como resultado de la cual se vio particularmente influenciada por el modelo biomédico. Influida por esa concepción, se instaló en la psiquiatría contemporánea una propuesta para abordar las enfermedades mentales que se apoya en una tríada de supuestos básicos articulados entre sí:

- En primer lugar, suponer que es posible una identificación objetiva y categorial de los trastornos o síndromes mentales por vía de una descripción "a-teórica" como se propone en la serie de los DSM (Diagnostic and Statistical Manual of Mental Disorders, de la American Psychiatric Association).

- En segundo lugar, si ese primer punto fuera posible, tratar de establecer una progresiva correlación biunívoca entre cada síndrome así definido y su fisiopatología cerebral.

- En tercer lugar, establecer una correlación entre dicha fisiopatología y su corrección farmacológica combinada con una psicoterapia basada en nuevos desarrollos de las teorías del aprendizaje. Presentándose como teorías psicológicas pasibles de verificación experimental, las corrientes cognitivocomportamentales han gozado de mayor interés en detrimento del psicoanálisis que apoya su validez en estudios de casos.

Aunque esta manera de presentar las cosas no cuenta con consenso de todos los psiquiatras y ha despertado críticas de diversos tipos, se perfila, sin embargo, en la especialidad como una potente propuesta paradigmática. Las razones de la misma no derivan solamente de su supuesta, y discutible, coherencia interna, sino, en gran medida, de una cantidad de factores externos a la disciplina (ideológicos, económicos y corporativos) que intentan imponerla.

En efecto, el peso cultural de algunos centros de investigación del hemisferio norte, la influencia de la industria farmacéutica, la caída del Estado benefactor, el crecimiento de los Servicios Gerenciados de Salud y cambios en las modas y hábitos culturales y en la subjetividad de la población, han figurado entre las principales causas de la prominencia de que goza actualmente esa expresión del modelo biomédico en 
psiquiatría (68).

\section{d) El diagnóstico en psiquiatría}

Toda concepción de la enfermedad mental, al tener que establecer sus límites y características específicas, genera conflictos éticos. El diagnóstico en psiquiatría, como en otras especialidades médicas, es el punto de partida del "acto psiquiátrico" y por lo tanto autoriza la intervención del psiquiatra, delimita el grupo de sujetos susceptibles a dicha intervención y propone los métodos de tratamiento a ser utilizados $(13,14)$.

En la medida en que el diagnóstico es un acto humano puede surgir, a partir de las cualidades del mismo, el peligro de "mal uso" o de "abuso", es decir, una aplicación de categorías diagnósticas a quienes no les corresponden, con el consiguiente riesgo de generar pérdida de la libertad personal y sometimiento de la víctima a ambientes y tratamiento psiquiátricos innecesarios; la posibilidad de quedar marcado por el estigma de ser un "enfermo mental" por un largo período o, incluso, para toda la vida; o hacerse pasible de una serie de consecuencias sociales y legales (declaración de incapacidad civil, curatelas, pérdida de diversos derechos, etc.) (13).

El diagnóstico psiquiátrico -modelado sobre el diagnóstico médico general- es un juicio de valor que pesa intensa y prolongadamente sobre el destino de quien lo recibe; no es una palabra neutra, puede contribuir a estructurar el futuro del caso clínico y hasta puede influir peyorativamente sobre el pronóstico.

La clínica psiquiátrica apoya su diagnóstico en una semiología específica, entendida como la identificación y discriminación entre conductas patológicas y normales (diferenciadas entre sí en forma categorial o dimensional). El inventario semiológico de la psiquiatría se conformó desde su nacimiento como especialidad médica a principios del siglo XIX, en el contexto de una cultura en permanente evolución que mediante diversas operaciones separó, más o menos radicalmente, las conductas "desviadas" de las "normales". La estructura de la semiología psiquiátrica es homomorfa con la de la medicina general. El diagnóstico se apoya en la semiología y precede, lógicamente, a la terapéutica. Al tiempo que se verifican constantemente en la clínica innumerables casos en los que el diagnóstico, la terapéutica y el pronóstico de personas con alteraciones mentales -calificadas como patológicas a partir de criterios semiológicos y valoraciones diagnósticas pertenecientes al tesaurus de la psiquiatría- respondieron correctamente según las previsiones de los especialistas, también aparecen no pocos casos en los que tal proceso no se desarrolla adecuadamente.

La observación más cuidadosa de este tipo de situaciones permite descartar factores de mala praxis (ignorancia, impericia, negligencia) e identificar otras razones de diagnósticos psiquiátricos erróneos. Estos pueden ocurrir: a) de forma intencionada en aquellos casos en los que el especialista administra conscientemente un diagnostico psiquiátrico convencional a quien no está afecto de enfermedad con fines no médicos tales como presión de una familia que pretende hospitalizar a un miembro problemático; presiones políticas para descalificar a un opositor, como ocurrió en el sistema soviético con el constructo de esquizofrenia leve o "tórpida" inventado por Snezhnevsky; por deseos del propio "paciente" cuando el diagnóstico puede eximirlo de un proceso judicial; para hallar una solución de tipo "coartada" y evitar un escándalo, etc.; o b) de forma no intencionada, más sutil e insidiosamente, y por lo tanto, más difíciles de identificar y suprimir. En esta última variedad el proceso diagnóstico sufre deformaciones en sus aspectos valorativos en función de necesidades, presiones y compromisos no técnicos que condicionan la conciencia del psiquiatra más o menos nebulosamente. El hecho de que los psiquiatras, por comodidad, se permitan funcionar como si no tuvieran ninguna conciencia de este hecho, o eludan la responsabilidad de fortalecerla, confiere a este tipo de diagnóstico erróneo un enorme interés ético (13).

En la mayoría de los diagnósticos erróneos no intencionados se pueden identificar diversos sesgos que deberían ser revisados desde la perspectiva bioética. Entre ellos se destacan los debidos a limitaciones propias de las herramientas de diagnóstico basadas en criterios explícitos y a las referencias teóricas en el establecimiento de nosologías. Pasaremos revista a los problemas más evidentes.

A pesar de la enorme difusión de los sistemas criteriológicos de diagnóstico (DSM, CIE 10) el diagnóstico psiquiátrico sigue siendo un proceso pasible de error y quien lo emite debe recordar sus limitaciones con humildad, mantenerse dispuesto a revisar sus decisiones diagnósticas y a admitir su caracter falible. En el mejor de los casos, los psiquiatras no superan a sus herramientas, $y$, para ser conscientes de sus propias limitaciones deben reconocer las de sus instrumentos diagnósticos. 
Es muy fácil equivocarse en el proceso diagnóstico en psiquiatría, en virtud de que no hay síntomas patognomónicos y ciertos criterios que pretendieron erigirse en discriminadores confiables para arribar con certeza a ciertos diagnósticos ("índice de comprensibilidad", "comportamiento peculiar", valoración por el psiquiatra de "su propia intuición", indicadores contratransferenciales, etc.) han demostrado su contenido subjetivo. En consecuencia, el diagnóstico psiquiátrico no puede "calcarse" sobre el de la medicina general que, frecuentemente y sobre todo en situaciones vitales de urgencia, adquiere la cualidad de acto instantáneo (por ejemplo: ACV, abdomen agudo, embolia pulmonar, etc.) sino que debe ser entendido como un proceso sometido, muy a menudo, a inconsistencias iniciales, con tendencia a cambios evolutivos y reorganizaciones clínicas (psíquicas) en horas o en días, bajo la eventual influencia de psicofármacos sintomáticos que van modificando de manera dinámica la valoración de la probable categoría diagnóstica (p. ej., casos de excitación psicomotriz, alteraciones sensoperceptivas bajo ansiedad aguda, etc.) .

Por otro lado, para abonar la necesidad de extremar la prudencia en el pronunciamiento diagnóstico, no deben olvidarse las conclusiones de Scheff según las cuáles los médicos en general consideran que un "Error tipo 2" (aceptar una hipótesis falsa) es menos peligroso que un "Error tipo 1" (rechazar una hipótesis cierta); estos dilemas pueden conducir a situaciones en las que se emitan diagnósticos psiquiátricos en personas que no presentan alteración de la salud mental (13) y viceversa. Este fenómeno acecha, particularmente, a los profesionales en formación que en nuestro medio, por fallas y carencias en las estructuras didácticas se ven forzados a emitir sus diagnósticos sin adecuada supervisión. En efecto, es más difícil diagnosticar la salud que una supuesta enfermedad, y no es raro ver que los psiquiatras que reciben a una persona en su consulta se sitúan subjetivamente en el campo observacional de la enfermedad, olvidando que su responsabilidad inicial como expertos es determinar primero si el motivo de consulta tiene calidad patológica o es una reacción adecuada a un estímulo intenso o a una crisis vital normal (como la tristeza del duelo, ciertos montos de ansiedad reactiva a situaciones económico-sociales, conductas adolescentes exageradas, etc.). Si bien es cierto que se podría explicar ese apresuramiento o confusión diagnóstica como simple impericia, también es cierto que la perspectiva ética podría extremarse hasta enjuiciar no solo, y cruelmente, a quienes se ven colocados al límite de sus posibilidades por las imperfecciones del sistema de salud en el que trabajan, sino al sistema de salud mismo que los expone a situaciones para las que nos los ha preparado adecuadamente.

Otro sesgo, que puede pasar inadvertido en la práctica corriente proviene del contexto sociocultural en el que tiene lugar el proceso diagnóstico. Los trastornos psiquiátricos suelen presentarse como transgresiones o desviaciones de las normas sociales y conductuales. De hecho estas son, a veces, los signos precoces o prodrómicos de enfermedad. Las referencias sociales del diagnóstico pueden devenir así en un problema, y el psiquiatra debe conocer con precisión innumerables y sutiles variables culturales y reconocer las conductas que obedecen a alguna motivación no patológica (tendencias artísticas, activismo social, simple excentricidad, pertenencia a otra cultura o subcultura). No son pocos los psiquiatras, en particular aquellos demasiado adheridos a sistemas de diagnóstico categoriales, que no asignan suficiente importancia a estos sesgos derivados de la dimensión situacional del diagnóstico.

Es elocuente en ese sentido que, en la encuesta realizada por la Asociación Mundial de Psiquiatría (WPA) y la Organización Mundial de la Salud (OMS) en 2011, entre 4887 psiquiatras de 44 países, en torno a su empleo de los sistemas de clasificación diagnóstica en el ejercicio clínico y las características idóneas de una clasificación de los trastornos mentales, con vistas a la revisión de la Clasificación Internacional de Enfermedades (cuya próxima versión será la CIE 11), un número significativo de los encuestados (particularmente de los países de Asia y América Latina) reportaron problemas con la aplicabilidad intercultural de las clasificaciones existentes (75).

Las teorías psicológicas y nosológicas que actúan como marcos referenciales no explicitados de los sistemas de diagnóstico psiquiátrico, tienen una influencia menos reconocida, pero comparable a la del contexto social y no menos operantes en la formulación de un diagnóstico. Casi todos los psiquiatras se guían por una o más teorías de la enfermedad mental (aunque este politeorismo es un tema que no desarrollaremos aquí merece ser mencionado por los sesgos epistemológicos que comporta, y en ciertos casos extremos es fuente de eclecticismos lindantes con la fantasía) que fundamentan las expresiones funcionales y prácticas de los sistemas nosológicos con los que operan. Los presupuestos y la especificidad del sistema al que adhieren para reconocer y definir 
una enfermedad mental influyen poderosamente sobre la manera en que abordan los especialistas al consultante, el enfoque que utilizan para evaluar y extraer conclusiones sobre el comportamiento de una persona, en esencia, su forma de "contemplar" a la persona. En la medida que el psiquiatra siga los criterios del sistema sin la adecuada flexibilidad y eleve los constructos clínicos del mismo a la categoría de entidades naturales, se puede caer en diagnósticos erróneos de apariencia sistemática y rigurosa. Aunque se ha pretendido minimizar ese sesgo en las últimas décadas por vía de las nosografías basadas en criterios explícitos, este peligro sigue existiendo. En los EE.UU., las ediciones más recientes del manual diagnóstico oficial, se han desplazado hacia un enfoque descriptivo y no teórico del diagnóstico de las enfermedades mentales, cuya etiología, en la mayoría de los casos, no ha llegado a establecerse.

En líneas generales, la serie de los DSM define los trastornos mentales describiendo sus rasgos clínicos más fácilmente identificables, advirtiendo enfáticamente que no clasifican individuos sino trastornos (síndromes) ni clasifican las enfermedades según etiologías, sino en función de sus características clínicas comunes; al mismo tiempo, alertan sobre la necesidad de tener más datos que los señalados en las categorías del manual para arribar a un diagnóstico integral. Desgraciadamente, los usos y costumbres tienden a hacer olvidar entre los usuarios del DSM estas sanas advertencias, lo cual conduce a una utilización simplificada y banal. Evidentemente, este riesgo es mayor cuanto menos específicos o polisémicos sean los síntomas señalados como indicadores de enfermedad mental, fenómeno que se puede ejemplificar más claramente en las enumeraciones de criterios que definen los denominados trastornos de la personalidad.

\section{La salud reducida a un bien de cambio}

"En el mundo actual, la comunidad científica arriesga perder su identidad y su vocación.

El ethos específico de la ciencia se torna cada vez más difícil de delimitar.

Hoy en día la ciencia y el gran capital están completamente asociados".

Sir Michael Atiyah, Presidente de la Royal Society de Londres, 1995.

Una ética orientada al respeto de los valores clásicos de la medicina debe tomar en consideración las condiciones sociales y económicas de la práctica médica $(69,76)$. Dentro del proceso universal de globalización se enfrentan dos teorías del orden social: la del Estado mínimo o mercantilista (propia de las corrientes neoliberales de la política y la economía) y la del Estado de bienestar o solidario (77). De ellas se desprenden modelos de Sistemas de Salud opuestos entre sí; no solamente por el tipo de gestión que caracteriza a cada uno de ellos, sino por las escalas de valores que entrañan, lo cual tiene implicancias bioéticas.

El modelo mercantilista aplica los criterios de la economía de mercado al ámbito de la salud. En este modelo, la salud se convierte exclusivamente en un bien de cambio, es decir, un producto destinado a la compra y la venta cuyo propósito es producir algún tipo de ganancia. Esta mercantilización acarrea desigualdades flagrantes en la entrega de la "mercancía" -la atención en salud- sin respetar el principio de igualdad de prestaciones, seleccionando a quienes pueden pagarla y otorgando al producto una calidad creciente de acuerdo al mayor poder adquisitivo del cliente. Quienes carezcan de ese poder, cuyo número aumenta en función del subdesarrollo de los diferentes países, deberán conformarse con una atención de calidad crecientemente menor o con la, generalmente, deficiente caridad estatal. Este modelo se concretiza en el sistema gerenciado de salud de progresiva extensión a nivel mundial.

En el extremo opuesto, el modelo solidario, basado en el respeto a los derechos sociales con garantía del Estado, se caracteriza por ajustarse a los siguientes principios: 1) toda persona tiene igual derecho al más amplio sistema de prestaciones básicas, 2) todas las personas deben gozar de la misma igualdad de oportunidades y 3 ) debe existir una desigualdad justa, es decir, dar más al que menos tiene.

La medicina solidaria es universal, equitativa y con control y prudencia en sus costos; sin recortes arbitrarios y basada en una asignación ética de recursos. Es un modelo que controla las sobre-prestaciones, la corrupción, la burocracia, y otras desviaciones de la correcta y honesta gestión del Estado (77). El respeto a los derechos sociales de los ciudadanos es condición para el desarrollo sano de la persona.

Esos dos modelos contrapuestos tienen efectos mayúsculos sobre el quehacer de los médicos, sobre sus condiciones de trabajo, sobre su gestión diagnóstica, sobre la toma de decisiones terapéuticas y por sobre todo sobre las características de la relación 
médico-paciente.

Como hemos señalado en otros trabajos, con la caída del Estado de bienestar, consecuencia del neoliberalismo económico, numerosos factores políticos, sociales y económicos tales como la crisis del Hospital Público y la mercantilización de los servicios médicos, la precariedad del empleo de los profesionales de la salud, el nuevo perfil de los hoy llamados usuarios (ex-pacientes), los intereses de sectores industriales como las industrias farmacéutica y de aparatología, etc., han adquirido una importancia determinante en las definiciones de lo que es un médico y de lo que es la medicina, y por ende, lo que es un psiquiatra y lo que es la misión de su especialidad.

Las decisiones éticas que el médico decida tomar estarán, en ese contexto, profundamente condicionadas. Las tensiones que puedan surgir son, con frecuencia, fuente de angustia, culpa, desánimo en el ejercicio profesional del lado del médico, y pérdida de excelencia en la prestación recibida por el paciente. El contexto socioeconómico condiciona profundamente las decisiones médicas y las consecuencias bioéticas de las mismas (69). La praxis psiquiátrica en sus aspectos socio-económicos, las características del sistema de salud en el cual ésta se desenvuelve y la estructura del pensamiento médico en la que se basa son factores condicionantes, cuando no determinantes, de las opciones éticas que se elijan.

Este complejo situacional contemporáneo debe tomarse en cuenta para la estructuración del currículo de medicina en los niveles de grado y de postgrado, y como analizador para considerar las decisiones éticas de la práctica médica cotidiana.

\section{CONCLUSIONES}

Sobre la base de los problemas enumerados cabe insistir en proposiciones ya enunciadas en trabajos anteriores, junto a otras resultantes de la presente reflexión que pueden aportar a los especialistas aspectos de interés para su estudio:

1. Tomar decisiones bioéticas implica hacerlo en el marco de conocimientos posibles que condicionarán a aquellas opciones o, más aún, podrían prefigurarlas. Si tales conocimientos provienen de una estructura de pensamiento biomédico reduccionista, las decisiones que se tomen estarán viciadas por el sesgo epistemológico provisto por la matriz de pensamiento utilizada. En consecuencia, para generar una psiquiatría premunida de un ética respetuosa del sufrimiento humano se debe partir de una crítica radical del modelo biomédico reduccionista en la especialidad. Y la única posibilidad de formular una crítica fundamentada a ese modelo se puede encontrar en la actualización de lo que ha dado en llamarse el modelo médico antropológico o sea una medicina de la persona, considerada ésta en su integridad e individualidad.

2. El diagnóstico psiquiátrico sufre una tensión ética: a) cuando se produce una "psiquiatrización" de la vida cotidiana de forma tal que conductas y vivencias son "etiquetadas" psiquiátricamente sin evaluarse las consecuencias, o bien cuando se produce una subjetivización del diagnóstico, es decir sin criterios de consenso. La "psiquiatrización", además de fomentar la difusión de los ya imprecisos límites de la enfermedad mental, puede dar lugar a la discriminación o estigmatización del sujeto o, en otros casos, privarlo del tratamiento idóneo; b) cuando el diagnóstico psiquiátrico se produce en contextos no sanitarios, donde entran en juego otros valores (en el ámbito judicial, por ejemplo) y donde el padecimiento de un trastorno puede suponer cursos de acción claramente diferentes en comparación con la ausencia del mismo; c) cuando la motivación del diagnóstico no es terapéutica ni evaluativa sino que responde a intereses individuales del paciente, del profesional, de familiares o, en ocasiones, de la propia sociedad que demanda agentes de control y/o d) cuando el diagnóstico, una vez realizado, presenta problemas en cuanto a su difusión (información al paciente y a terceros) o es empleado para limitar la autonomía de la persona.

Santander y colaboradores $(14,16,18,19)$, sugieren algunos principios que, con ciertos complementos (41), pueden ser utilizados para enfrentar los problemas descritos: emitir un diagnóstico con criterios clínicos razonados y razonables, utilizando los medios disponibles, clínicos, e instrumentales (tests) y basándose en la congruencia de los hallazgos con los criterios internacionalmente aceptados, matizados y completados con cuantos datos complementarios se estimen oportunos; disponer de una formación adecuada en los conocimientos que deben manejarse para la elaboración de un diagnóstico, contar con la supervisión de colegas más avezados y/o compartir el trabajo en el seno de un equipo de Salud Mental. Debe quedar claro que el diagnóstico no es sino un medio que orienta hacia el tratamiento; es además urgente no emitir diagnósticos dudosos o personales, que suelen 
conllevar una mayor estigmatización, es mejor aplazar un diagnóstico que tener que revisar continuamente una situación clínicamente poco clara, cuidar exquisitamente la confidencialidad y no prestarse en ningún caso a situaciones en las que el diagnóstico pueda utilizarse de forma interesada o equivoca fuera del ámbito evaluativo o terapéutico.

3. Una ética basada en el rescate de valores antropológicos en la concepción de la medicina, debe considerar las condiciones sociales y económicas de la práctica médica.

El respeto a los derechos sociales de los ciudadanos es condición básica para la preservación de la salud mental de las personas, y modela el accionar de los psiquiatras tanto a nivel de sus decisiones técnicas como de los valores bioéticos que las mismas vehiculizan. La práctica de una psiquiatría sustentada por una crítica bioética se ve impedida o dificultada por los sistemas de salud mercantilistas, el asistencialismo y la beneficencia privada o estatal.

\section{Correspondencia:}

Juan Carlos Stagnaro.

Moreno 1785, 5to. Piso.

Ciudad Autónoma de Buenos Aires. Argentina.

Correo electrónico: jcstagnaro@gmail.com

\section{Conflictos de interés:}

El autor declara no tener conflictos de interés.

\section{REFERENCIAS BIBLIOGRÁFICAS}

1. Espejo D, Castilla A. (Editores). Bioética en las Ciencias de la Salud. Jaén: Asociación Alcalá; 2001.

2. Hottois G. El paradigma bioético. Barcelona: Anthropos; 1991.

3. Desviat M, Gonzalez C, Gonzalez A, et al. Actitudes éticas y práctica clínica de los psiquiatras españoles. Psiquiatría Pública. 1999; 11: 147-156.

4. Gracia D. Prólogo. En: Santander F (Coordinador). Ética y Praxis psiquiátrica. Madrid: Asociación Española de Neuropsiquiatría. Estudios 23; 2000.

5. Musto DF. Perspectiva histórica. En: Bloch S, Chodoff P, Green SA (Editores). La ética en psiquiatría. Primera edición española. Madrid: Triacastela; 2001.p. 19-34.

6. Ruiz M, Espejo $M^{a} D$. Historia y definición de la Bioética. En: Sánchez J, Sánchez J. El consentimiento informado en psiquiatría. Madrid: Editorial Díaz de Santos; 2002. p. 81-91.
7. Sgreccia E. Justificación epistemológica, fundamentación del juicio bioético y metodología de la investigación en bioética. En: Sgreccia E. Manual de Bioética. México DF: Editorial Diana; 1996. p. 51-79.

8. Morera B, Santander F. La perspectiva ética en la praxis psiquiátrica: ¿Dónde estamos? Archivos de Psiquiatría. 2001; 64 (4): 289-293.

9. Outomuro D. La relación médico paciente desde una perspectiva bioética. Rev Fund Fac de Medicina. 2001; 11 (42): 23-26.

10. Beauchamp TL. Los fundamentos filosóficos de la ética en psiquiatría. En: Bloch S, Chodoff $\mathrm{P}$, Green S.A (Editores). La ética en psiquiatría. Primera edición española. Madrid: Triacastela; 2001.p. 35-56.

11. Alby JM, Gayda M, Vacola G, et al. Ethique en psychiatrie. Paris,France : Elseveier.1995.p.1-8.

12. Association Française de Psychiatrie. Colloque : Principes et Éthique du soin en psychiatrie. Psy Fr. $2007 ; 3$.

13. Reich W. El diagnóstico psiquiátrico como problema ético. En: Bloch S, Chodoff P (editores). La ética en psiquiatría. Primera edición española. Madrid: Triacastela; 2001.p. 189-211.

14. Santander F, Morera B, Retolaza A, et al. El proceso diagnóstico. En: Santander F. (coordinador). Ética y praxis psiquiátrica. Madrid: Asociación Española de Neuropsiquiatría. Estudios/23. 2000. p. 114121.

15. Sánchez J. El consentimiento informado en psiquiatría. Madrid: Editorial Díaz de Santos; 2002. p. 81-91.

16. Santander F, Morera B, Retolaza A, et al. El consentimiento informado y la valoración de la capacidad para decidir. En: Santander F. (coordinador). Ética y praxis psiquiátrica. Madrid: Asociación Española de Neuropsiquiatria; 2000. p. 54-75.

17. Morera B. Secreto médico y práctica psiquiátrica. En: Calcedo A. Secreto médico y protección de datos sanitarios en la práctica psiquiátrica. Madrid: Panamericana. 2000. p. 121-145.

18. Santander F, Morera B, Retolaza A, et al. Confidencialidad. En: Santander F. (Coordinador) Ética y praxis psiquiátrica. Madrid: Asociación Española de Neuropsiquiatría; 2000. p. 42-54.

19. Santander F, Morera B, Retolaza A, et al. El proceso terapéutico. En: Santander F (Coordinador) Ética y praxis psiquiátrica. Madrid: Asociación Española de Neuropsiquiatría. Estudios/23; 2000. p. 151171.

20. Álamo C. La información farmacoterapéutica en el paciente psiquiátrico. En: Amarilla M, Alamo C; ASEDEF. El Consentimiento en la utilización de fármacos. Alcalá de Henares: Universidad de 
Alcalá de Henares. 2000. p.47-68.

21. Brown P, Pantelis C. Aspectos éticos del tratamiento farmacológico. En: Bloch S, Chodoff P, Green SA. La ética en psiquiatría. Primera edición española. Madrid: Triacastela; 2001. p. 235-262

22. Castilla A. Implicaciones éticas de la participación del paciente psiquiátrico en su tratamiento. Bioética y Ciencias de la Salud. 2000; 4(1):10-11.

23. Fernández P, Cañabate E, Salas L, et al. Aspectos éticos de la desinstitucionalización en el enfermo mental crónico. Cuadernos de Bioética. 1999; 1040: 661-667.

24. Holmes J. Aspectos éticos de las psicoterapias. En: Bloch S, Chodoff P, Green SA. La ética en psiquiatría. Primera edición española. Madrid: Triacastela; 2001. p. 217-234.

25. Peele R, Chodoff P. La ética del tratamiento involuntario $\mathrm{y}$ la desinstitucionalización. En: Bloch S, Chodoff P, Green SA (editores). La ética en psiquiatría. Primera edición española. Madrid: Triacastela; 2001. p. 409-413.

26. Szmukler G. Ética en la psiquiatría comunitaria. En: Bloch S, Chodoff P, Green SA (editores). La ética en psiquiatría, Primera edición española. Madrid: Triacastela; 2001. p. 341-357

27. Arechederra JJ. Informes periciales en psiquiatría. Madrid: You \& Us; 2001.

28. Gutheil TG. Ética y psiquiatría forense En: Bloch S, Chodoff P, Green SA (Editores). La ética en psiquiatría. Primera edición española. Madrid: Triacastela; 2001. p. 323-339.

29. Santander F, Morera B, Retolaza A, et al. Actividad ante terceros: peritajes, testimonios e informes. En Santander F (coordinador) Ética y praxis psiquiátrica. Madrid: Asociación Española de Neuropsiquiatría. Estudios/23; 2000. p. 201-209.

30. Mendiburu L. Etica y psiquiatría. Tesis Master en Bioética, Cordova, España. Instituto de Estudios Bioéticos, 2003.

31. Bloch S, Chodoff P, Green SA. Introducción. En: Bloch S, Chodoff P, Green SA. (Editores). La ética en psiquiatría. Primera edición española. Madrid: Triacastela; 2001. p. 13-17.

32. Desviat M. Ética y psiquiatría (Internet). Interpsiquis. 2001; (2). (Citado el 7 de febrero del 2013). Disponible en: http//www.psiquiatria.com/ imprimir. ats? 2670>

33. Losoviz AI, Vidal DA, Bonilla A. Bioética y Salud Mental. Buenos Aires: Akadia; 2006.

34. Megía J, Moreno JJ. (Coord.). Salud Mental y Bioética, Reflexiones desde una perspectiva disciplinar. Valencia: Servicio de Salud Mental, Dirección General de Asistencia Sanitaria, Generalitat Valenciana, Conselleria de Sanitat. 2013.

35. Fantin JC, Fridman P. Bioética Salud Mental y Psicoanálisis. Buenos Aires: Polemos; 2009.

36. Escrig M, Vergara C, Megía MJ. Salud mental y bioética desde el análisis métrico de la literatura científica. En: Megía J, Moreno J (Coordinador), Salud Mental y Bioética, Reflexiones desde una perspectiva disciplinar. Valencia: Servicio de Salud Mental, Dirección General de Asistencia Sanitaria, Generalitat Valenciana, Conselleria de Sanitat; 2013.

37. Ramos S. El consentimiento informado en la esquizofrenia: revisión del concepto «dignidad». En: Boladeras M. (Editores) ¿Qué dignidad? Filosofía, Derecho y práctica sanitaria. Capellades: Proteus; 2010. p. 217-230.

38. Kallert TW, Glöckner M, Onchev G, et al. Proyecto EUNOMIA sobre la coacción en psiquiatría: diseño del estudio y datos preliminares. World Psychiatry. 2005; 3: 3 .

39. Ramos S. La toma de decisiones compartidas en pacientes con esquizofrenia: cuestiones médicas y éticas. Dilemata. Revista Internacional de Éticas Aplicadas. 2012; 10: 263-277.

40. Simón P. La capacidad de los pacientes para tomar decisiones. Revista de la Asociación Española de Neuropsiquiatría. 2008; 2: 327-350.

41. Cañas F, Santander F, Morera B. Confidencialidad en Psiquiatría. En: Confidencialidad en la práctica psiquiátrica Guía breve. Madrid: ASEDEF; 2002. p. 33-63.

42. Santander F. El respeto a la confidencialidad en el contexto sanitario. Revista Norte de Salud Mental. 2003; 16:15-19.

43. Appelbaum P. Psychiatric advance directives and the treatment of committed patients. Psychiatric Services. 2004; 55(7):751-753.

44. Gorrotxategi M, Romero J. Voluntades psiquiátricas: Una herramienta terapéutica. Norte de salud mental. 2012; 10(42):11-17.

45. Joshi K. Psychiatric advance directives. Journal of Psychiatric Practice. 2003; 9:303-306.

46. Ramos S. Las decisiones por representación en la esquizofrenia: aspectos médicos, legales y éticos. En: Boladeras M. Bioética: la toma de decisiones. Capellades: Proteus; 2011. p. 385-398.

47. Srebnik D, Rutherford L, Peto T, et al. The content and clinical utility of psychiatric advance directives. Psychiatric Services. 2005; 56(5):592-498.

48. Swanson J, Swartz M, Elbogen E, et al. Psychiatric advance directives and reduction of coercive crisis interventions. J Ment Health. 2008; 17(3):255-267.

49. Van Dorn R, Swartz M, Elbogen E, et al. Clinicians' attitudes regarding barriers to the implementation of psychiatric advance directives. Administration and Policy in Mental Health and Mental Health Services Research. 2006; 33(4):449-460.

50. Wilder Ch, Elbogen E, Moser L, Swanson J, Swartz, M. Medication preferences and adherence among individuals with severe mental illness and psychiatric advance directives. Psychiatric Services. 2010; 
61(4):380-385.

51. Bloch S, Pargiter R. Códigos éticos en psiquiatría. En: Bloch S, Chodoff P, Green S.A. (editores) La ética en psiquiatría. Primera edición española. Madrid: Triacastela; 2001. p. 89-107.

52. Herranz G. El código de ética y deontología médica. Rev Clin Esp. 2003; 203 (2): 79-84.

53. Cortina A. Neuroética y neuropolítica. Sugerencias para la educación moral. Madrid: Tecnos; 2011.

54. Echarte L. Cómo pensar sobre el cerebro. Hacia una definición de neuroética. Medicina y persona. 2004; 48 (1): 38-41.

55. Evers K. Towards a philosophy for neuroethics. An informed materialist view of the brain might help to develop theoretical frameworks for applied neuroethics. EMBO Rep. 2007;8 S48-51.

56. Fuchs T . Ethicals issues in neurosciences. Curr Opin Psych. 2006; 19 (6):600-7.

57. Giménez Amaya JM, Sánchez-Migallón S. De la Neurociencia a la Neuroética. Narrativa científica y reflexión filosófica. Pamplona: EUNSA; 2010.

58. Northoff G. What is neuroethics? Empirical and theoretical neuroethics. Current Opinion in Psychiatry. 2009, 22:1-5.

59. Suárez M. Psiquiatría y neuroética. VERTEX Revista Argentina de Psiquiatría. 2013; 24 (109): 6875.

60. Lanzilota A. Comités de Bioética. VERTEX Revista Argentina de Psiquiatría. 2007;18 (75): 382-386.

61. Conti NA. Una experiencia en curso. Reportaje a los miembros del Comité de Bioética del Hospital José T. Borda (G.C.B.A.). VERTEX Revista Argentina de Psiquiatria. 2007; 18 (75): 387-390.

62. Stagnaro JC. Los psiquiatras y los hospitales psiquiátricos: del asilo a la comunidad". VERTEX Revista Argentina de Psiquiatría, 2006; 11(65): 2834.

63. Castel R. Prólogo. En: Alvarez F. Miserables y locos. Medicina mental y orden social en la España del siglo XIX. Barcelona: Tusquets; 1983.

64. Kraut JA. Salud Mental. Tutela Jurídica. Santa Fe: Rubinzal-Culzoni, 2006. p. 437-487.

65. Kraut JA. Pacientes mentales y derecho privado. Salud mental. Tutela Jurídica. Santa Fe: RubinzalCulzoni; 2006. p.187-254.
66. Organización Panamericana de la Salud. Declaración de Caracas. Caracas, Venezuela: Conferencia sobre la Reestructuración de los Servicios Psiquiátricos en América Latina; 1990.

67. Thurin JM. Tableau de la recherche psychiatrique en France. Pour la Recherche, Bulletin de la Fédération Française de Psychiatrie. 1995; (5): 5-8.

68. Stagnaro JC. Biomedicina o medicina antropológica. VERTEX Revista Argentina de Psiquiatría. 2002; 13 (47):19-26.

69. Stagnaro JC. La formación de especialistas en psiquiatría hoy: un desafío epistemológico. VERTEX Revista Argentina de Psiquiatría. 2000; 11 (S1).

70. Roa A. La deshumanización de la medicina y Responsabilidad de médico y paciente ante la enfermedad a lo largo del siglo XX. En: Ética y bioética. Santiago de Chile: Andrés Bello; 1998, p. 142-163.

71. Laín P. Antropología médica. Barcelona: Salvat; 1984.

72. Meeroff M. Medicina integral, Salud para la comunidad. Buenos Aires: Catálogos; 1999.

73. Gil C. Introducción a la medicina antropológica. Caracas: Universidad Central de Venezuela; 1974.

74. Meeroff M. Definición de médico integral, Claves. 1999; 9(15/16): 21-26.

75. Reed GM, Mendonça J, Esparza P, Saxena S, Mario M. Encuesta mundial de WPA-WHO sobre las actitudes de los psiquiatras hacia la clasificación de los trastornos mentales. World Psychiatry. 2011; 10:118-131.

76. Neuchloz SM. La medicina como ciencia y como actividad social. Buenos Aires: Losada; 1944.

77. Lazzari AH. Bioética de la distribución de recursos en salud. Desarrollos en Psiquiatría Argentina. 1997; 2 (4) 63-68.

Recibido: 10/12/2012

Aceptado: 16/01/2013 